\title{
In Silico Characterization and Screening for Inhibitors of Sdia: A Protein Involved in Quorom Sensing in Escherichia Coli
}

\author{
Joseph Christina Rosy, Shalini Mohan, Praseetha Sivakumar, Shakti Chandra Vadhana \\ Marimuthu, Krishnan Sundar
}

\begin{abstract}
Bacteria use quorum sensing as a way of inter and intra- species communication. Quorum sensing was found to be important for bacteria for various processes including establishing an infection through virulence and biofilm formation. This is mediated autoinducers, which are usually produced by one group of bacteria and recognized by another group through a response regulator protein. LuxR is a response regulator protein first discovered in Vibreo fischeri and it recognizes autoinducers produced by the same species of bacteria. E. coli also has a response regulator called SdiA which is a homolog of LuxR, originally found to be involved in transcription and cell division. SdiA was later reported to regulate quorum sensing by binding to autoinducers called Acyl Homoserine Lactones (AHLs). SdiA is also reported to be involved in enhancing the multidrug resistance and virulence in pathogenic $E$. coli. Though many studies elaborate the functional aspects of SdiA, sequence and structural level analysis of this protein is missing in the literature. The current work aims at the in silico analysis and targeting of SdiA with structural analogs of AHLs. 7 compounds were found to be promising molecules to inhibit quorum sensing in E. coli.
\end{abstract}

Keywords : AHLs, SdiA, Quorum sensing.

\section{INTRODUCTION}

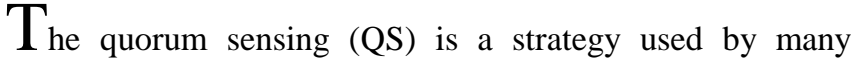
bacteria that controls gene expression in a cell density dependent manner. QS is a cell to cell communication process in which bacteria synthesize, recognize and respond to extracellular signaling molecules. QS mediates many processes in bacteria including bioluminescence, secretion of virulence factors, formation of biofilm, etc. QS mainly involves the production of chemical signaling molecules,

Revised Manuscript Received on December 16, 2019.

* Correspondence Author

Joseph Christina Rosy, Department of Biotechnology, Kalasalingam Academy of Research and Education, Krishnankoil, Tamilnadu. Email: j.christinarosy@klu.ac.in

Shalini Mohan, Department of Biotechnology, Kalasalingam Academy of Research and Education, Krishnankoil, Tamilnadu. Email: shallushals01@gmail.com

Praseetha Sivakumar, Department of Biotechnology, Kalasalingam Academy of Research and Education, Krishnankoil, Tamilnadu. Email: praseethasiva24@gmail.com

Shakti Chandra Vadhana Marimuthu, Department of Biotechnology, Kalasalingam Academy of Research and Education, Krishnankoil, Tamilnadu. Email: chandravadhana16@gmail.com

Krishnan Sundar, Department of Biotechnology, Kalasalingam Academy of Research and Education, Krishnankoil, Tamilnadu. Email: sundarkr@klu.ac.in followed by the sensing of them by the QS regulators to generate a response. The signaling molecules are referred as the autoinducers (AIs).

AIs are produced by bacteria at basal levels and their concentration increases with growth. Then these molecules diffuse through bacterial membranes and enter the cells. When reaching an optimal concentration, the autoinducer molecules interact with response regulators present inside bacterial cells and activate them, which in turn can alter gene expression. As this phenomenon happens in a cell-density-dependent manner, it is termed as quorum sensing [1-4]. QS requires AIs and response regulator proteins. AIs bind to response regulator proteins to activate it. The activated regulatory protein binds to promotor regions of genes inducing transcription of the genes [3]. Many QS systems were described based on the type of AIs and regulator proteins involved. The three main QS systems are i) LuxR/AI-1 system ii) LuxS/AI-2 system, iii) AI-3/epinephrine/norepinephrine system. E. coli utilizes all the three QS systems. In Lux R/AI-1 system, E. coli detect autoinducers from other bacteria. In LuxS/AI-2 system E. coli participate in intra and inter species signaling. During the AI-3 /epinephrine/ norepinephrine system E. coli recognize self produced autoinducer, or signals by other bacteria, or human stress hormones epinephrine and norepinephrine. The most intensely studied AI-1s in Gram negative bacteria are the AHLs [1]. E. coli can produce AI-2 molecules but not AI-1 (AHLs) [5].

Autoinducer molecules are synthesized by LuxI and its homologues by transferring a fatty acid chain from an acylated acyl carrier protein to $S$-adenosylmethionine thereby releasing the AHL and methylthioadenosine [6]. Bacteria that contain LuxI gene or its homologues can produce AHL. AHL structure contains a homoserine lactone ring linked to a fatty acid side chain. Each species of bacteria produces a distinct AHL molecule. For example, the length of acyl chain may contain four to 18 carbons, and acyl chains differ in the degree of saturation. Further, the AHL may differ at the third carbon of the acyl chain and contain a hydrogen, hyrdroxyl, carbonyl, or oxo group [4, 7, 8]. E. coli lacks LuxI and thus do not produce AHLs. But E. coli recognizes AHLs produced by other bacteria through a LuxR homolog known as SdiA [9]. SdiA detects a much broader range of AHLs than other LuxR homologs [10]. In E. coli, SdiA was reported to regulate biofilm formation, motility and indole production [11-13].

\section{Published By:}


SdiA mediated quorum sensing is used by $E$. coli $\mathrm{K}-12$ for virulence gene expression [14] and AHL analogues are reported to inhibit the expression of virulence genes [15]. Thus it is apparent that inhibiting SdiA would be a novel way to control the virulence of $E$. coli. Interference with quorum sensing is being considered as one of the new strategies that can be regarded as an alternative to antibiotics [16]. Although SdiA has reported for its molecular mechanisms its complete structural and functional properties were unexplored. Hence, the current work aims to perform in silico sequence and structure analysis of SdiA and also screening for binders of SdiA that could potentially useful as regulators of quorum sensing.

\section{METHODS}

\section{A. Compounds}

Four AHLs which are most commonly produced in bacteria were selected from the literature (Table 4). Compounds with structural similarity with these 4 AHL molecules were retrieved using ChemMine tool [17]. 83 ligands which are similar to 4 AHL molecules were retrieved and used in this study.

\section{B. Structure of SdiA}

The crystal structure of SdiA from E. coli was retrieved from Protein Data Bank (PDB ID: 4Y15). It contains 246 amino acids. The structure is complexed with 3-oxo-C6-homoserine lactone.

\section{Computational Details}

All computations were carried out using a computer with 3GB RAM and Intel Core 2 duo E7500 processor $(2.93 \mathrm{GHz})$. Molecular docking studies were performed with AutoDock 4.2 running in Debian version 9. Result analysis was performed with Discovery Studio Visualizer client 4.0.

\section{Sequence analysis of SdiA}

Sequences of SdiA of E. coli K-12 and LuxR of $V$. fischeri were retrieved from Uniprot database and pair wise alignment was performed to check their identity and similarity. Also multiple sequence alignment was performed with the SdiA and LuxR family of proteins from other organisms.

\section{E. Phylogenetic Analysis}

Evolutionary history of SdiA was analyzed by constructing a Phylogenetic tree from the protein sequences of SdiA and LuxR homologs of various organisms using MEGA X [18].

\section{F. Physicochemical Properties}

Various physicochemical properties were predicted for SdiA using ExPASy-ProtParam tool [19] and subcellular localization was predicted using CELLO2GO webserver [20].

\section{G. Structural analysis of SdiA}

Structural motifs and domains present in the SdiA were analyzed using InterPro. Structure alignment of SdiA with LuxR was performed using TM-align [21] in order to understand the insights of structural similarity between these proteins.

\section{H. Molecular Docking Studies}

The protein and all the 83 ligands were prepared for docking using the graphical user interface of AutoDock tools. The preparation involved adding all hydrogen atoms to the proteins, which is a step necessary for calculation of partial atomic charges. Water molecules and heteroatoms were removed from the protein molecule [22].

By using the graphical user interface of AutoDock tools, a 3D grid box was generated to embed the protein and, Grid parameters were set. Grid maps were calculated by running AutoGrid 4. Docking parameters were set by the docking wizard of AutoDock Tools [23]. Conformation search was performed using Lamarckian Genetic Algorithm which runs for 100 cycles [24]. The binding energies for each conformation of the ligand with the proteins were determined by running AutoDock 4 [25].

Analysis of docking was performed by using the graphical user interface of AutoDock Tools and Discovery Studio Client 4.0. Binding energies of each conformation of docked compounds were noted and the best conformation was chosen based on the binding energy and number of hydrogen bonds that they form with the protein. Various types of interactions between ligand and receptor such as hydrogen bonds, hydrophobic interactions, Van der Waals and electrostatic interactions were visualized.

\section{RESULTS}

\section{A. Sequence Analysis of SdiA}

Sequence level identity and similarity between the SdiA of E. coli and LuxR of V. Fischeri was found to be $39 \%$ and $49 \%$ respectively. This indicates that though $\mathrm{SdiA}$ was reported to be a homolog of LuxR, it has a low sequence similarity with LuxR. Mulltiple sequence alignment of SdiA and LuxR of various organisms was preformed and it was found that the residues in the $\mathrm{C}$ - terminal regions of proteins were conserved (data not shown).

\section{B. Phylogenetic Analysis}

Phylogenetic analysis was performed with the SdiA and LuxR sequences of various organisms in order to track the evolutionary history of SdiA. (Figure 1).

\section{Physicochemical Properties}

The physicochemical properties such as amino acid composition, theoretical pI, extinction coefficient and instability index were obtained and listed in Table 1 . The amino acid composition of this protein is shown in Table 2. The number of leucine residues in the protein is high next to alanine. Number of hydrophobic residues is high in this protein and so the aliphatic index was found to be higher.

\section{Structural Analysis of SdiA}

SdiA contains two domains as analyzed by InterPro, one in the N-terminal and the other in the C-terminal. The $\mathrm{N}$-terminal domain is 'Winged helix-like DNA-binding domain' and the N-terminal domain is the 'Transcription factor LuxR-like, autoinducer-binding domain'. The details of these domains are presented in Table 3. Structures of LuxR and SdiA were aligned using TM-align and the

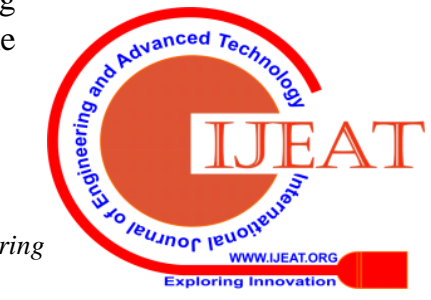


superimposed regions are shown in Figure 4.

\section{E. Molecular Docking Studies}

The ligands (n-83) were docked against the crystal structure of SdiA using AutoDock 4.2. The binding energy of each ligand and number of hydrogen bonds that they make with the protein were recorded (data not shown).

The four AHL molecules and the natural ligand that is present in the crystal structure were also docked. The binding energies of the 4 AHL molecules are presented in Table 4. The compounds showing lower binding energy than the 4 AHL molecules were considered to be top binders.

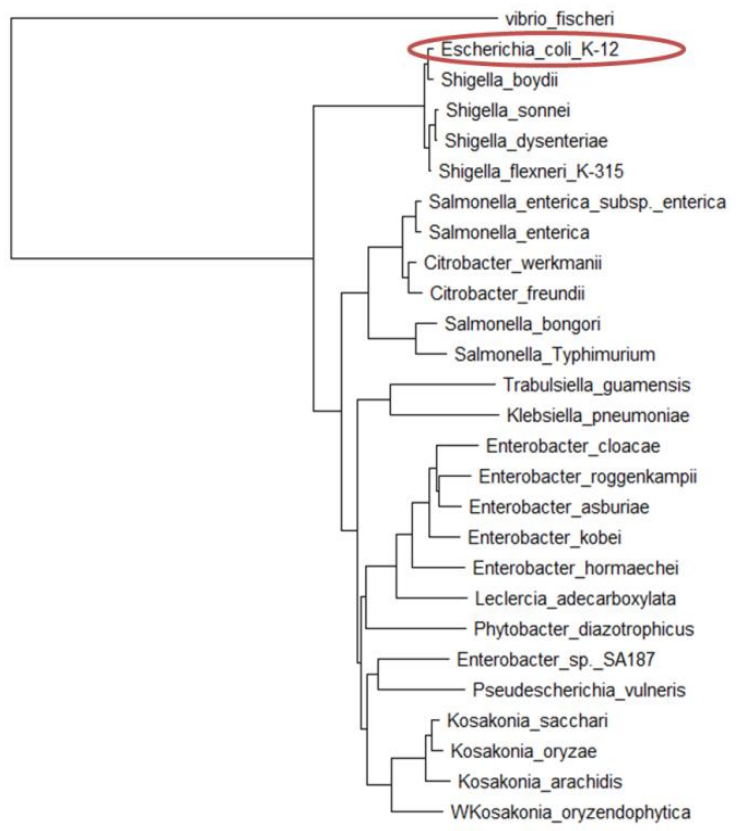

0.10

Fig. 2. Phylogenetic Tree showing the evolutionary relationship of selected SdiA and LuxR from different organisms

Table 1: Physicochemical Properties of SdiA

\begin{tabular}{|l|l|}
\hline Molecular weight & 29182.49 \\
\hline Theoretical pI & 6.60 \\
\hline $\begin{array}{l}\text { Total number of } \\
\text { negatively charged } \\
\text { residues (Asp + Glu) }\end{array}$ & 29 \\
\hline $\begin{array}{l}\text { Total number of } \\
\text { positively charged } \\
\text { residues (Arg + Lys) }\end{array}$ & 27 \\
\hline Estimated half-life & $\begin{array}{l}30 \text { hours (mammalian } \\
\text { reticulocytes, in vitro) } \\
>20 \text { hours (Yeast, in vivo) } \\
>10 \text { hours (Escherichia coli, in } \\
\text { vivo) }\end{array}$ \\
\hline $\begin{array}{l}\text { Instability index } \\
\text { This classifies the protein as } \\
\text { unstable. }\end{array}$ \\
\hline Aliphatic index & 81.09 \\
\hline $\begin{array}{l}\text { Grand average of } \\
\text { hydropathicity } \\
\text { (GRAVY) }\end{array}$ & -0.413 \\
\hline
\end{tabular}

Table 2: Amino acid composition of SdiA

\begin{tabular}{|l|l|l|}
\hline Amino acid & $\begin{array}{l}\text { Number of } \\
\text { residues in } \\
\text { the protein }\end{array}$ & Percentage \\
\hline Ala (A) & 21 & $8.5 \%$ \\
\hline Arg (R) & 16 & $6.5 \%$ \\
\hline Asn (N) & 13 & $5.2 \%$ \\
\hline Asp (D) & 8 & $3.2 \%$ \\
\hline Cys (C) & 3 & $1.2 \%$ \\
\hline Gln (Q) & 14 & $5.6 \%$ \\
\hline Glu (E) & 21 & $5.5 \%$ \\
\hline Gly (G) & 6 & $2.4 \%$ \\
\hline His (H) & 11 & $4.4 \%$ \\
\hline Ile (I) & 11 & $4.4 \%$ \\
\hline Leu (L) & 27 & $10.9 \%$ \\
\hline Lys (K) & 11 & $4.4 \%$ \\
\hline Met (M) & 12 & $4.8 \%$ \\
\hline Phe (F) & 12 & $4.8 \%$ \\
\hline Pro (P) & 11 & $4.4 \%$ \\
\hline Ser (S) & 14 & $5.6 \%$ \\
\hline Thr (T) & 11 & $4.4 \%$ \\
\hline Trp (W) & 5 & $2.0 \%$ \\
\hline Tyr (Y) & 10 & $4.0 \%$ \\
\hline Val (V) & 11 & $4.4 \%$ \\
\hline Pyl (O) & 0 & $0.0 \%$ \\
\hline Sec (U) & 0 & \\
\hline & & $0.0 \%$ \\
\hline & 11 & \\
\hline
\end{tabular}

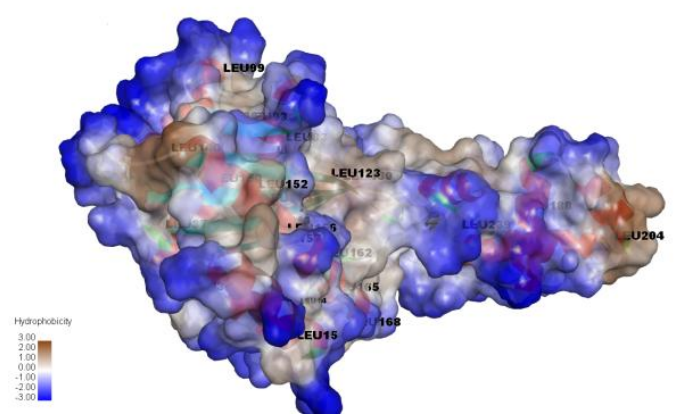

Fig. 3. Hydrophobicity map of SdiA with highlighted Leucine residues

Table 3: Domains present in SdiA

\begin{tabular}{|l|l|l|}
\hline \multicolumn{1}{|c|}{$\begin{array}{l}\text { Name of the } \\
\text { domain }\end{array}$} & \multicolumn{1}{|c|}{$\begin{array}{l}\text { Description } \\
\text { no } \\
\text { acids }\end{array}$} \\
\hline $\begin{array}{l}\text { Winged helix-like } \\
\text { DNA-binding } \\
\text { domain superfamily }\end{array}$ & $\begin{array}{l}\text { Winged helix DNA-binding } \\
\text { proteins contain a related } \\
\text { winged helix-turn-helix } \\
\text { DNA-binding motif where } \\
\text { the "wings are small } \\
\text { beta-sheets. The winged helix } \\
\text { motif consists of two wings, } \\
\text { three alpha helices (H1, H2, } \\
\text { H3) and three beta-sheets (S1, } \\
\text { S2, S3) arranged in the order } \\
\text { H1-S1-H2-H3-S2-W1-S3-W2 }\end{array}$ & \\
\hline
\end{tabular}




\begin{tabular}{l|l|l|} 
Transcription-factor & $\begin{array}{l}\text { This domain binds N-acyl } \\
\text { homoserine lactones (AHLs). }\end{array}$ & 145 \\
LuxR-like, & In most cases, binding of AHL \\
autoinducer-bindin & by this N-terminal domain & \\
g domain & $\begin{array}{l}\text { leads to unmasking of the } \\
\text { DNA-binding domain, } \\
\text { allowing it to bind DNA and } \\
\text { activate transcription. }\end{array}$ & \\
\end{tabular}

Based on this, the cut off value for top binders were set as $-7.61 \mathrm{kcal} / \mathrm{mol}$, which is the lowest binding energy value showed by the AHL molecule, C8. Out of the 83 compounds docked, 7 compounds showed binding energy of less than $-7.61 \mathrm{kcal} / \mathrm{mol}$. (Table 5)

The compounds Beta- D- gluco- hexopyranosyluronic acid$(1->3)-$

$2-$ acetamido-2-deoxy-beta-D-gluco-hexopyranosyl- (1->4)beta- D- gluco- hexopyranosyluronic acid(1->3)-2-acetamido-2-deoxy- beta- D- gluco-hexopyranose (cpd a), Etelcalcetide, Cyclosporine, Clindamycin and Beta-D- gluco- hexopyranosyluronic acid-(1->3)-2-acetamido-2-deoxy-hexopyranosyl-(1->4)-bet a-D-gluco-hexopyranosyluronic acid- (1->3)- 2- acetamido2- deoxy- hexopyranose (cpd b), Clindamycin Phosphate and Glutathione were the top binders. Binding interactions of the top binder cpd a with SdiA is given in Figure 5. The amino acids interacting with these top 7 compounds are given in Table 5.

Table 4: List of AHL molecules selected for the study and their binding energies with SdiA

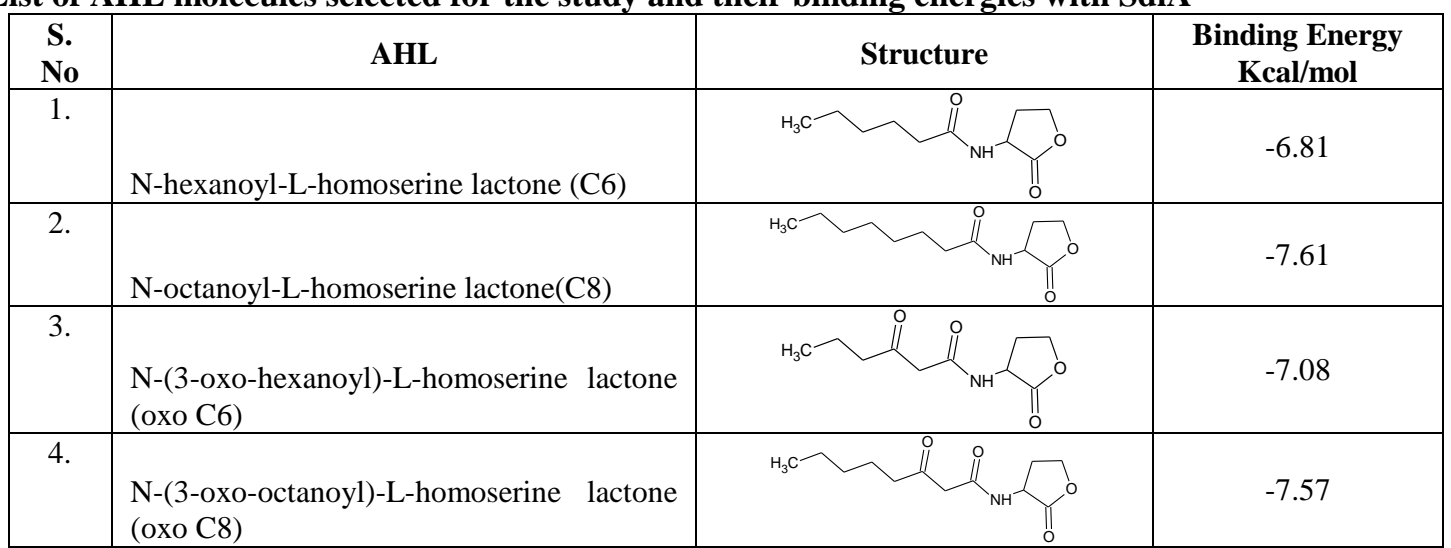

Table 5: Amino acids of SdiA interacting with top 7 binders

\begin{tabular}{|l|l|l|l|}
\hline Rank & Compound & $\begin{array}{l}\text { Binding Energy } \\
\text { Kcal/mol }\end{array}$ & Interacting amino acids \\
\hline 1 & Cpd a & -9.88 & Pro51, Arg54, Pro55, Lys56, Asn223 \\
\hline 2 & Etelcalcetide & -9.56 & $\begin{array}{l}\text { Pro51, Arg54, Pro55, Lys56, Val57, Gln72, } \\
\text { Pro225, Gln229 }\end{array}$ \\
\hline 3 & Cyclosporine & -9.14 & $\begin{array}{l}\text { Pro81, Leu83, Pro85, Glu86, Asn87, Phe88, } \\
\text { Ser89, Leu99, Tyr122, Met124, Leu130 }\end{array}$ \\
\hline 4 & Clindamycin & -8.52 & Arg54, Val57, Leu77, Ala78, Pro81, Leu83 \\
\hline 5 & Cpd b & -8.5 & $\begin{array}{l}\text { Asn127, Met166, Thr175, Met178, Lys182, } \\
\text { Lys185, Arg189, Leu239, Ile240, His241, } \\
\text { His242 }\end{array}$ \\
\hline 6 & $\begin{array}{l}\text { Clindamycin } \\
\text { Phosphate }\end{array}$ & -8.28 & Met163, Met166, Lys182, Lys185, \\
\hline 7 & Glutathione & -7.95 & Lys182, Lys185, Arg189, His242 \\
\hline
\end{tabular}

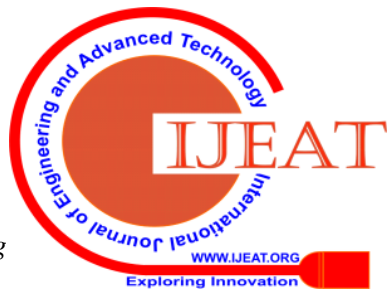




\section{DISCUSSION}

Bacteria interact with their community through a group of small signaling molecules known as autoinducers. This process called as quorum sensing, involves a group of individual organisms to an environmental response. Earlier reports underscore the importance of quorum sensing in virulence and biofilm formation in bacteria [26-29].

The autoinducers are either produced by one species and recognized by the other or produced and recognized by the same species. For recognizing the autoinducers the bacteria need a response regulator protein. LuxR is such a response regulator found in many bacteria initially discovered in $V$. fischeri. E. coli do not produce autoinducers but it can recognize the autoinducers called AHLs through a LuxR homolog called SdiA. SdiA belongs to the LuxR family of transcriptional regulators that induce ftsQAS locus in cell division [13]. SdiA enhances multidrug resistance by stimulating efflux pumps in $E$. coli [11]. Thus SdiA could be a potential target towards pathogenic strains of E. coli such as uropathogenic E. coli and Enteropathogenic E. coli [30].

Though many studies have found out the molecular mechanism of SdiA function, no reports are available on sequence and structural level comparison of LuxR with SdiA. The current study involves in silico characterization of SdiA and screening for inhibitors using molecular docking studies. Various physicochemical parameters of SdiA were analyzed and was found that SdiA is rich in hydrophobic residues especially leucine (Figure 3). This could contribute to higher thermal stability of the protein [31].

The pairwise sequence alignment of SdiA and LuxR shows that they share less percentage similarity but the multiple sequence alignment of sequences of LuxR family of proteins shows that the homology between these proteins is due to the conserved residues present in the $C$-terminal region of the protein. This is further confirmed by the structure alignment which shows that the two domains, DNA binding domain and AHL binding domain are overlapping in Lux-R and SdiA. The winged helix-like DNA-binding domain consists of two wings (W1, W2), three alpha helices $(\mathrm{H} 1, \mathrm{H} 2, \mathrm{H} 3)$ and three beta-sheets (S1, S2, S3) arranged in the order H1-S1-H2-H3-S2-W1-S3-W2. All these wings, helices and sheets are overlapping in LuxR and SdiA (Figure 4).

Molecular docking studies were performed with $83 \mathrm{AHL}$ analogs as analog-based inhibition is one of the promising strategies in designing new drugs. Seven top binders were found to have lower binding energy than the AHL molecules. The amino acids Arg54, Lys182, Lys185 and His242 are found to be interacting with 3 or more top binding compounds, which suggest that these amino acids might be essential for binding. These top compounds can further be studied as inhibitors of quorum sensing using in vitro studies and can be developed into potential drugs

\section{REFERENCES}

L.C. Antunes and R. B. Ferreira, "Intercellular communication in bacteria" Crit Rev Microbio., 2009, Vol 135, pp 69-80

2. B. L. Bassler, and R. Losick, "Bacterially speaking” Cell, vol. 125, pp. 237-246.

3. W. C. Fuqua, S. C. Winans, and E. P. Greenberg, "Quorum sensing in bacteria: the LuxR-LuxI family of cell density-responsive transcriptional regulators" J Bacteriol, 1994, vol . 176, pp. 269-275.
4. W. C. Fuqua and E. P. Greenberg, "Listening in on bacteria: acylhomoserine lactone signalling”, Nat Rev Mol Cell Biol, 2002, vol. 3, pp. 685-695.

5. M. G. Surette, B. L. Bassler, ".Quorum sensing in Escherichia coli and Salmonella typhimurium”, Proc Natl Acad Sci USA., 1998, vol. 95 pp. 7046-7050.

6. A. L. Schaefer, D. L. Val, B. L. Hanzelka, J. E. Cronan Jr, and E. P. Greenberg, "Generation of cell-to-cell signals in quorum sensing: acyl homoserine lactone synthase activity of a purified Vibrio fischeri LuxI protein", Proc Natl Acad Sci USA, 1996, vol. 93(18), pp. 9505-9509.

7. M. M. Marketon, M. R. Gronquist, A. Eberhard, J. E. González "Characterization of the Sinorhizobium meliloti $\sin R / \sin I$ locus and the production of novel $\mathrm{N}$-acyl homoserine lactones", J Bacteriol, 2002, vol. 184, pp.5685-5695.

8. N. A. Whitehead, A. M. L. Barnard, H. Slater, N. J. Simpson, GPC Salmond, "Quorum-sensing in Gram-negative bacteria" FEMS Microbiol Rev, 2001, vol. 25, pp. 365-404.

9. S. Henikoff, J. C. Wallace, and J. P. Brown, "Finding protein similarities with nucleotide sequence databases", Methods. Enzymol., 1990, vol 183, pp. 111-132.

10. L. Steindler, V. Venturi, "Detection of quorum-sensing N-acyl homoserine lactone signal molecules by bacterial biosensors", FEMS Microbiol Lett, 2007, vol. 266, pp.1-9.

11. J. Lee, A. Jayaraman, T. K. Wood, "Indole is an inter-species biofilm signal mediated by SdiA”, BMC Microbiol, 2007, vol. 7, pp. 42.

12. J. Lee, X. S. Zhang, M. Hedge, W. E. Bentley, A. Jayaraman, T. K.. Wood, "Indole cell signaling occurs primarily at low temperatures in Escherichia coli” ISME J., 2008, vol. 2, pp. 1007-1023

13. J. Lee, T. Maeda, S. H. Hong, T. K. Wood, "Reconfiguring the quorum-sensing regulator SdiA of Escherichia coli to control biofilm formation via indole and $N$-acylhomoserine lactone", Appl Environ Microbiol, 2009, vol. 75, pp. 1703-1716.

14. D. M. Sitnikov, J. B. Schineller, and T. O. Baldwin, "Control of cell division in Escherichia coli: regulation of transcription of ftsQA involves both RpoS and SdiAmediated autoinduction", Proc Natl Acad Sci USA, 1996, vol. 93, pp. 336-341.

15. K. Kanamaru, K. Kanamaru, I. Tatsuno, T. Tobe and C. Sasakawa, "Regulation of virulence factors of enterohemorrhagic Escherichia coli O157:H7 by self-produced extracellular factors", Biosci Biotechnol Biochem, 2000, vol. 64, pp. 2508-2511

16. R. G. Finch, D. I. Pritchard, B. W. Bycroft, P. Williams and G. S. A. B. Stewart, "Quorum sensing: a novel target for anti-infective therapy", J Antimicrob Chemother, vol. 42, pp. 569-571.

17. Backman, W. H. Tyler, Yiqun Cao, and Thomas Girke. "ChemMine tools: an online service for analyzing and clustering small molecules", Nucleic Acids Res, 2011, vol. 39.suppl_2, pp. W486-W491.

18. S. Kumar, G. Stecher, M. Li, C. Knyaz, and K. Tamura, "MEGA X: Molecular Evolutionary Genetics Analysis across Computing Platforms", Mol Biol Evol, 2018, vol. 35, pp. 1547-1549.

19. E. Gasteiger, C. Hoogland, A. Gattiker, S. Duvaud.,M. R. Wilkins, R. D. Appel, A. Bairoch, "Protein Identification and Analysis Tools on the ExPASy Server" (In) John M. Walker (ed): The Proteomics Protocols Handbook, Humana Press ,2005, pp. 571-607

20. C. S. Yu, C. W. Cheng, W. C. Su, K. C. Chang, S. W. Huang, J. K. Hwang, and C. H. Lu, "CELLO2GO: A Web Server for Protein subCELlular LOcalization Prediction with Functional Gene Ontology Annotation", PLoS ONE , 2014, vol. 9(6), ppe99368.

21. Y. Zhang and J. Skolnick, "TM-align: A protein structure alignment algorithm based on TM-score", Nucleic Acids Res, 2005, vol. 33: pp. 2302-2309.

22. R.S. Yedidi, Z. Liu Z, I. A. Kovari, P. M. Woster, L. C. Kovari, "P1 and P1' para-fluoro phenyl groups show enhanced binding and favorable predicted pharmacological properties: Structure-based virtual screening of extended lopinavir analogs against multi-drug resistant HIV-1 protease", J Mol Graph Model, 2014, vol. 47, pp. 18-24.

23. R. Haribalaganesh, J. Christina Rosy, S. Rohita, C. Aishwarya, S Brintha, S. Rahul, and K. Sundar. "Antiviral drugs against Ebola: A structure based virtual screening approach", Indian J Biotech, 2018, vol. 17, pp. 176-184

24. G. M. Morris, R. Huey, W. Lindstrom, M. F. Sanner, R. K. Belew, D. S. Goodsell and A. J. Olson, "Autodock4 and AutoDockTools4 automated docking with selective receptor flexiblity", J. Comput Chem, 2009, vol. 30, pp.2785-2791. 
25. S. Cosconati, S. Forli, A. L. Perryman, R. Harris, D. S. Goodsell and A. J. Olson, "Virtual screening with AutoDock: theory and practice", Expert Opin Drug Discovery, 2010, vol.5: 597-607

26. M. B. Miller, B. L. Bassler, "Quorum sensing in bacteria", Annu Rev Microbiol, 2001, vol. 55, pp. 165-199.

27. C. M. Waters, B. L. Bassler, "Quorum sensing: Cell-to-cell communication in bacteria", Annu Rev Cell Dev Biol, 2005, vol. 21, pp. 319-346.

28. S. B. van Bodman, J. M. Willey, S. P. Diggle, "Cell-cell communication in bacteria: United we stand", J Bacteriol, 2008, vol. 190, pp. 4377-4391.

29. G. M. Dunny, B. A. Leonard, "Cell-cell communication in Gram-positive bacteria", Annu Rev Microbiol, 1997, vol. 51, pp. 527-564.

30. V. Ravichandiran, K. Shanmugam, K. Anupama, S. Thomas, and A. Princy, "Structure-based virtual screening for plant-derived SdiA-selective ligands as potential antivirulent agents against uropathogenic Escherichia coli”, Eur J Med Chem, 2012, vol. 48, pp. 200-205.

31. S. Idicula-Thomas and P. V. Balaji, P. V, "Understanding the relationship between the primary structure of proteins and its propensity to be soluble on overexpression in Escherichia coli", Protein Sci, 2003, vol. 14(3), pp. 582-592.

\section{AUTHORS PROFILE}

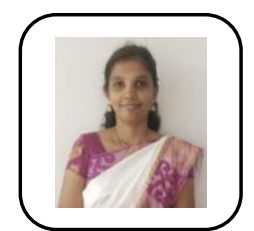

Christina Rosy, Joseph is currently working as an Assistant Professor in the Department of Biotechnology, Kalasalingam Academy of Research and Education. She has completed her Masters' in Biotechnology and pursuing $\mathrm{PhD}$ on 'Iron and Virulence'.

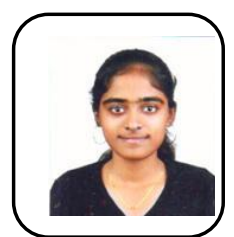

Shalini Mohan is a final year student of B. Tech Biotechnology, Kalasalingam Academy of Research and Education. Her research interest is on Bioinformatics and Drug Design.

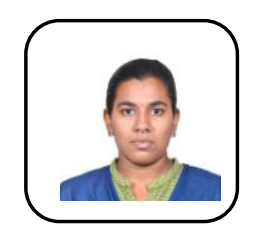

Praseetha Sivakumar is a final year student of B. Tech Biotechnology, Kalasalingam Academy of Research and Education. Her research interest is on Immunology and Drug Design.

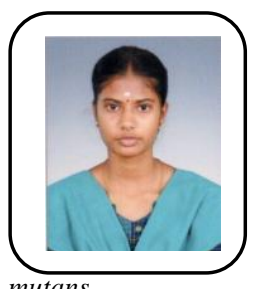

Shakti Chandra Vadhana Marimuthu graduated with a Master's degree in Microbiology and is currently a Research Fellow in the Department of Biotechnology, Kalasalingam Academy of Research and Education, working in the field of infectious diseases. Her current work focuses on quorum sensing and iron sequestration mechanisms in gram positive oral pathogen, Streptococcus

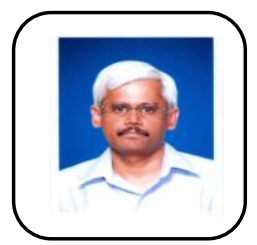

Dr Krishnan Sundar is a Professor of Biotechnology at Kalasalingam Academy of Research and Education. He received his Ph.D. degree in Microbiology from Madurai Kamaraj University. His research interests include target identification and drug design using in silico tools. 